\title{
The Brazilian research contribution to knowledge of the plant communities from Antarctic ice free areas
}

\author{
ANTONIO B. PEREIRA ${ }^{1}$ and JAIR PUTZKE ${ }^{2}$ \\ ${ }^{1}$ Instituto Nacional de Ciência e Tecnologia Antártico de Pesquisas Ambientais/INCT-APA, Universidade Federal do Pampa/ \\ UNIPAMPA, Campus São Gabriel, Av. Antonio Trilha, 1847, 93000-000 São Gabriel, RS, Brasil \\ ${ }^{2}$ Instituto Nacional de Ciência e Tecnologia Antártico de Pesquisas Ambientais/INCT-APA, Universidade de Santa Cruz do Sul/ \\ UNISC, Av. Independência, 2293, Bairro Marechal Rondon, 96815-900 Santa Cruz do Sul, RS, Brasil
}

Manuscript received on June 1, 2011; accepted for publication on September 12, 2011

\begin{abstract}
This work aims to summarize the results of research carried out by Brazilian researchers on the plant communities of Antarctic ice free areas during the last twenty five years. Since 1988 field work has been carried out in Elephant Island, King George Island, Nelson Island and Deception Island. During this period six papers were published on the chemistry of lichens, seven papers on plant taxonomy, five papers on plant biology, two studies on UVB photoprotection, three studies about the relationships between plant communities and bird colonies and eleven papers on plant communities from ice free areas. At the present, Brazilian botanists are researching the plant communities of Antarctic ice free areas in order to understand their relationships to soil microbial communities, the biodiversity, the distribution of the plants populations and their relationship with birds colonies. In addition to these activities, a group of Brazilian researchers are undertaking studies related to Antarctic plant genetic diversity, plant chemistry and their biotechnological applications.
\end{abstract}

Key words: Scientific publication, Botany, South Shetlands Island, Antarctica.

\section{INTRODUCTION}

The importance of the study of plant species found in the ice-free areas of Antarctic were related with the environmental monitoring, as a source for to evaluate the global changes. Climate changes is expected to have a major impact on the terrestrial biota of the Antarctic. Studies suggested that increasing temperatures and greater water availability could extend the active season, increase development rates and reduce the life cycle leading to altered the species distribution (Turner and Marshall 2011).

Correspondence to: Antonio Batista Pereira

E-mail: anbatistape@gmail.com
One of the first expeditions to the South Pole was carried out by the French explorer Lozier Bouvet in 1739. His discovery was named as Bouvet Island, but he had no scientific interest on the region. The first systematic botanic studies were carried out by J. Torrey in 1823, when he describes the Usnea fasciata. J. Eights was the first scientist to collect lichens, mosses, sea algae and grasses, between 1829-1830 (Putzke and Pereira 2001).

The Brazilian introduction in the Antarctica began in 1882, when the astronomer Luiz Antonio Cruls, on request of the French Academy of Science, held several observation about the Venus 
passage across the sun. Hundred years after, in 1982, starts the effective Brazilian participation through the first Brazilian Antarctic expedition on board of the oceanographic support ship Barão de Teffé (Souza 2008).

The plant communities in the Antarctic ice free areas are comprised of populations of three flowering plants (of which two are natives): Deschampsia antarctica Desv. (Poaceae) and Colobanthus quitensis (Kunth) Bartl (Caryophyllaceae) and Poa annua L. (Poaceae), accidently introduced in the 1980s (Olech 1996). The latter is native to Europe and was found growing in the vicinity of the Polish Arctowski Station, Admiralty Bay, King George Island, South Shetlands. For bryophytes 110 mosses species and 22 liverworts species are cited. More than 360 species of lichens are recognized (Øvstedal and Lewis Smith 2004). There are two species of macroscopic algae growing in and at the surroundings of bird colonies (Pereira 2004).

The Antarctic flora has been studied continuously since its discovery, but only a few efforts have been made to really explore the species richness and relationship in different icefree areas. The South Shetland Archipelago has $540 \mathrm{~km}$ of islands distributed over a SW to NE line, located in the northern part of the Antarctic Peninsula (ca. $160 \mathrm{~km}$ at north). King George Island is the largest one and the main area used for research bases in Antarctica. Argentina, Brazil, Chile, China, Poland, Russia, South Korea and Uruguay have permanently keep open bases on this island. Other countries have seasonal summer stations on different parts of this island, which demonstrates that human impact are strong on King George Island relative to other areas in the Maritime and Continental Antarctic.

This work aims to present the results of research carried out by Brazilian researchers on the plant communities of Antarctic ice free areas at the last twenty years in the South Shetland islands.

\section{MATERIALS AND METHODS}

Since 1988 field work has been carried out in different areas of the South Shetland Islands: Elephant Island (Stinker Point), King George Island (Admiralty Bay, Fildes Peninsula, Turret Point and Lions Rump), Nelson Island (Rip Point), Deception Island (Figures 1A-B, Figure 2). Phytosociological approaches were used to study the plant succession cover in the four islands.

Species identification were carried out with help of specialized literature, such Øvstedal and Lewis Smith (2001, 2004, 2009), Putzke and Pereira (2001) and Ochyra et al. (2008). The mosses names was up to date using the plant names database available in W3Tropicos web server (www.tropicos.org). For Bryaceae species was use the recent nomenclature following (Spence 2005, 2007). The lichen names was revised using the index fungorum database (www.indexfungorum. org) and most recent literature for antarctic lichens (Øvstedal and Lewis Smith 2001, 2004, 2009). The exsiccates of the specimens collected in Antarctica were deposited principally in the HCB Herbarium, ULBRA Herbarium, Botanical Garden of Rio de Janeiro Herbarium (RB) and HBEI (UNIPAMPA).

\section{RESULTS AND DISCUSSION}

The Brazilian contribution to the study of plant communities in Antarctic ice free areas, began as the foundation of the Brazilian Antarctic Program that performed the first Scientific Antarctic Expedition in Austral-Summer 1982/1983. In the first and second expeditions only oceanology studies were carried out, including cartographic and marine biology survey. Research on the land ecosystems was made possible after the inauguration of the Brazilian Antarctic Station in 1984, named Comandante Ferraz. Research on terrestrial plants began in the Austral Summer 1985 and 1986, with the study of lichens chemistry published by Xavier-Filho (1986, 1988, 1989, 

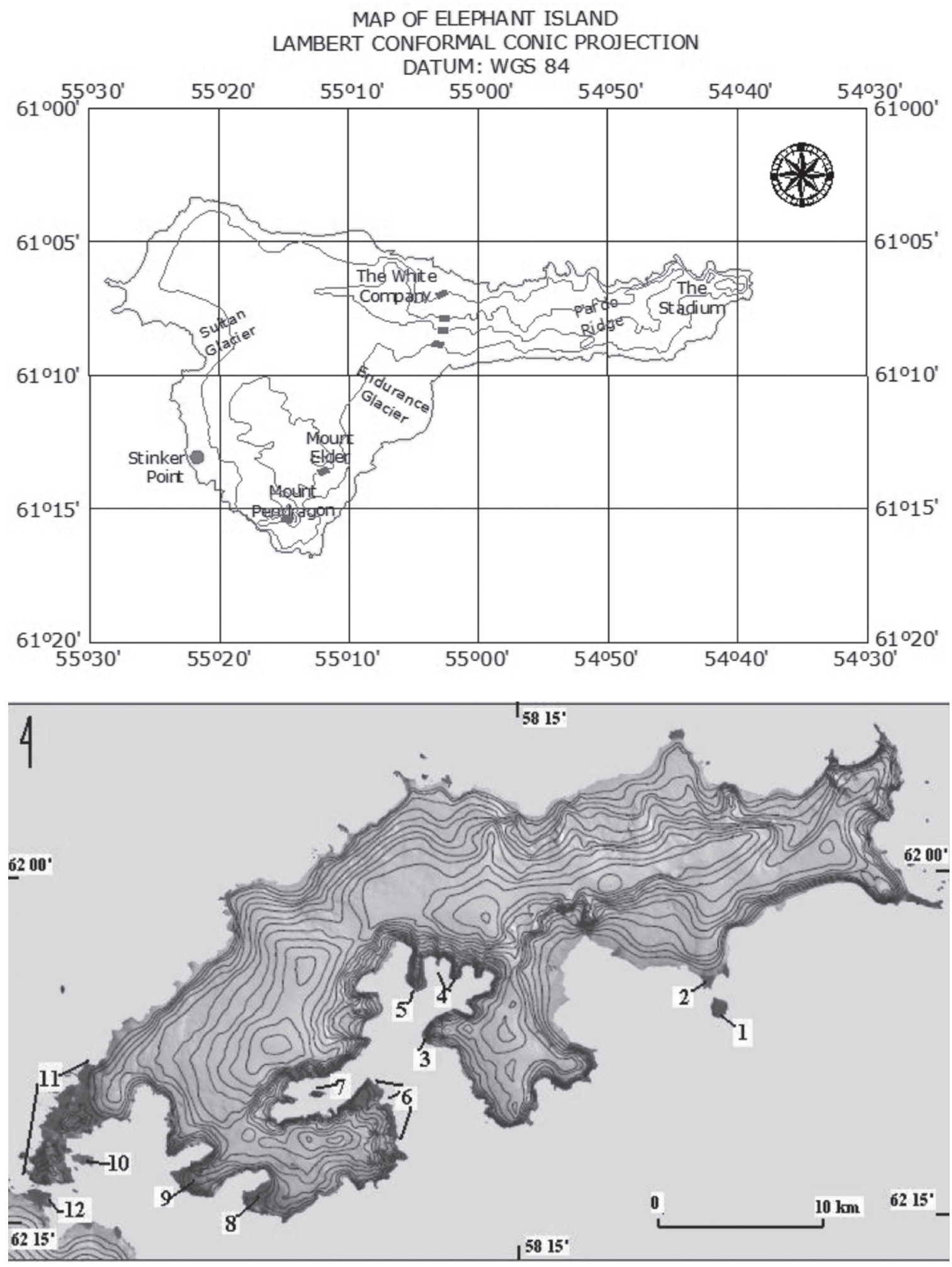

Figure 1 - Maps of Islands visited by the group: Elephant island (upper part), King George and Nelson Islands (middle) and Deception Island (down). 1 - Penguin Island. 2 - 11 King George Island: 2- Turret Point; 3- Hennequin point; 4-Ullman Point and Stenhouse Bluff; 5- Keller Peninsula; 6- Region from Thomas Point to Telefone Point; 7- Dufaiel Island; 8- Potter Peninsula; 9- Barton Peninsula; 10- Ardley Island; 11- Fildes Peninsula; 12 - Nelson Island (Rip Point). 


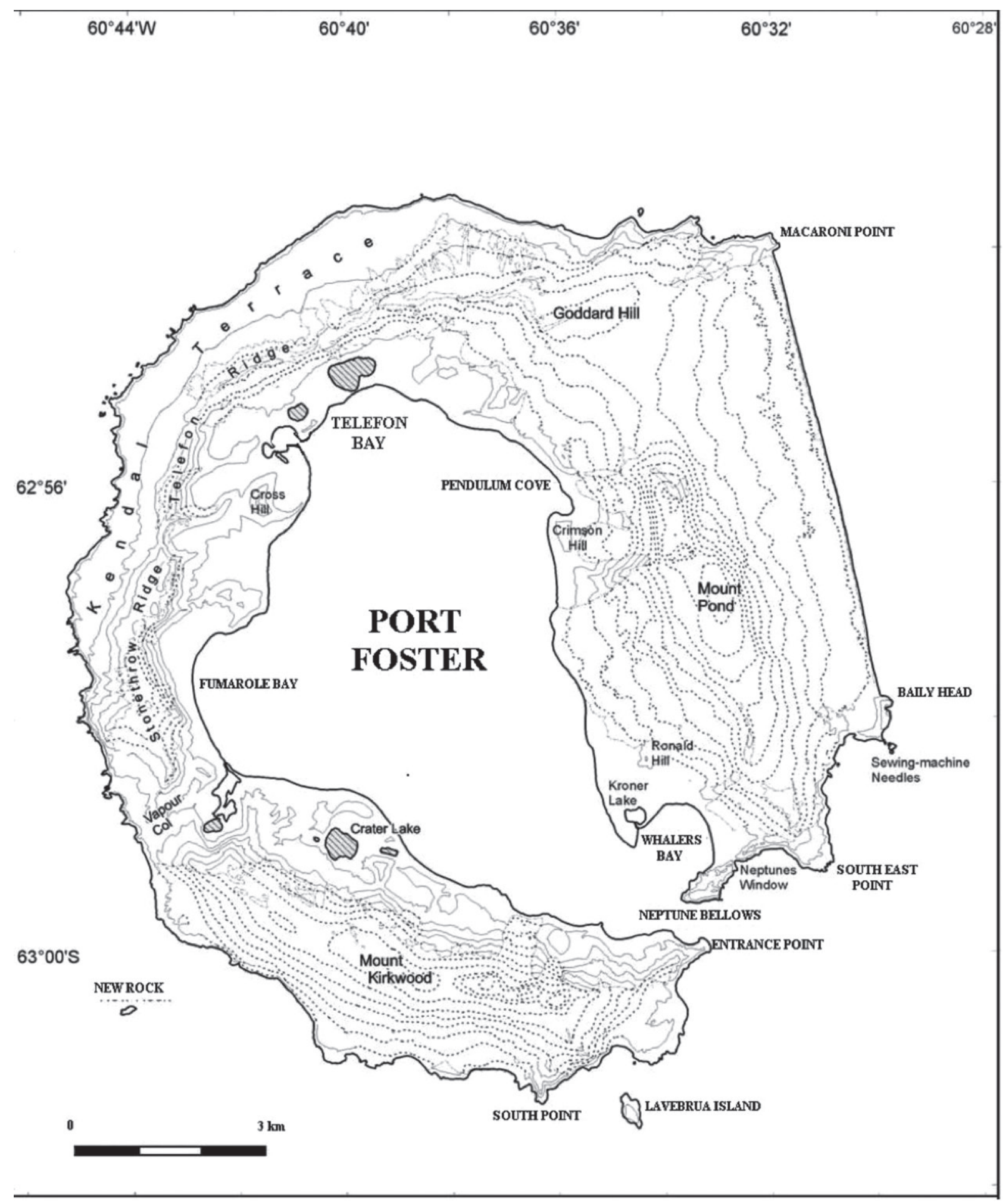

Figure 2 - Deception Island map, where all ice free areas were visited.

1990) and Czeczuga and Xavier-Filho (1987, 1988), describing beta-carotenoid, natural products and the antimicrobial activity of several lichen species.
Since 1988 the Brazilian Antarctic Program botanists initiated the study of plant communities in melting areas. Early works are published on 
the plant taxonomy, including first moss species list for King George Island (Putzke and Pereira 1990), as summarize in Table I, as well as the new records for Usnea trachycarpa (Pereira 1990), and other taxonomical studies (Putzke and Pereira 1996, 1998a) (see plant checklist in the present paper). More recently the first book on Antarctic Mosses (Putzke and Pereira 2001), was published a contribution on diatoms of Deception Island (Sramkova et al. 2007) and the first record of a myxomycete species in Antarctica (Putzke et al. 2004). Were also carried out studies on plant biology as Elster et al. 2008, Gonçalves et al. 2008, Kovácik et al. 2004, Martins et al. 2004 and Kovácik and Pereira 2001 detail information on the Mastodia tesselata (Hook. f. \& Harv.) Hook. f. \& Harv. lichenization processes. In addition, studies regarding the relationships between plant communities and bird colonies were published by Pereira et al. (1990a, b) and Sander et al. (2004). The photoprotective effect of extracts of Deschampsia. antarctica, Colobanthus quitensis, and Polytrichum juniperinum Hedw., against UVB. They attributed their photoprotective properties to several molecules, such as flavonoids and carotenoids, that absorb UV, act as antioxidants, and stimulate DNArepair processes (Kappel Pereira et al. 2004, 2009). Several plant community studies were carried out, such as Pereira (2004) (the first phytosociological survey for Antarctica), Pereira and Putzke (1994), Pereira et al. (2007, 2008, 2009), Putzke and Pereira (1998b), Victoria et al. (2004, 2006, 2009a, b) and Victoria and Pereira (2007). These studies will be useful for environmental impact evaluations in the ice-free areas by the Brazilian Antarctic Program in the future.

TABLE I

Moss species collected in the four South Shetland Islands by Brazilian botanist.

\begin{tabular}{|c|c|c|c|c|}
\hline MOSSES & Elephant & $\begin{array}{c}\text { King } \\
\text { George }\end{array}$ & Nelson & Deception \\
\hline \multicolumn{5}{|l|}{ AMBLYSTEGIACEAE } \\
\hline $\begin{array}{l}\text { Cratoneuropsis relaxa ssp. minor (Hook. f. \& Wilson) Ochyra [=Orthotheciella } \\
\text { varia (Hedw.) Ochyra] }\end{array}$ & $\mathrm{X}$ & $X$ & & \\
\hline Sanionia uncinata (Hedw.) Loeske & $\mathrm{X}$ & $\mathrm{X}$ & $\mathrm{X}$ & $\mathrm{X}$ \\
\hline Sanionia georgicouncinata (Müll Hal.) Ochyra \& Hedenas & $\mathrm{X}$ & $\mathrm{X}$ & $\mathrm{X}$ & $X$ \\
\hline Warnstorfia fontinaliopsis (Müll. Hal.) Ochyra & $\mathrm{X}$ & $\mathrm{X}$ & $\mathrm{X}$ & $\mathrm{X}$ \\
\hline Warnstorfia sarmentosa (Wahlenb.) Hedenas & $\mathrm{X}$ & $\mathrm{X}$ & $\mathrm{X}$ & \\
\hline \multicolumn{5}{|l|}{ ANDREAEACEAE } \\
\hline Andreaea depressinervis Cardot & $\mathrm{X}$ & $\mathrm{X}$ & $\mathrm{X}$ & $\mathrm{X}$ \\
\hline Andreaea gainii Cardot & $\mathrm{X}$ & $\mathrm{X}$ & $\mathrm{X}$ & $\mathrm{X}$ \\
\hline Andreaea regularis Müll. Hal. & $\mathrm{X}$ & $\mathrm{X}$ & $\mathrm{X}$ & $\mathrm{X}$ \\
\hline \multicolumn{5}{|l|}{ BARTRAMIACEAE } \\
\hline Bartramia patens Brid. & $\mathrm{X}$ & $\mathrm{X}$ & $\mathrm{X}$ & $\mathrm{X}$ \\
\hline Conostomum magellanicum Sull. & & $\mathrm{X}$ & $\mathrm{X}$ & \\
\hline Philonotis polymorpha (Müll. Hal.) Kindb. (=Bartramia polymorpha Müll. Hal.) & & & & $\mathrm{X}$ \\
\hline \multicolumn{5}{|l|}{ BRACHYTHECIACEAE } \\
\hline Brachythecium austrosalebrosum (Müll. Hal.) Paris & $\mathrm{X}$ & $\mathrm{X}$ & & \\
\hline Brachythecium austroglareosum (Müll. Hal.) Paris & & $\mathrm{X}$ & $\mathrm{X}$ & \\
\hline Brachythecium subpilosum (Hook. f. \& Wilson) A. Jaeger & & $\mathrm{X}$ & & \\
\hline
\end{tabular}


TABLE I (continuation)

\begin{tabular}{|c|c|c|c|c|}
\hline MOSSES & Elephant & $\begin{array}{l}\text { King } \\
\text { George }\end{array}$ & Nelson & Deception \\
\hline \multicolumn{5}{|l|}{ BRACHYTHECIACEAE } \\
\hline $\begin{array}{l}\text { Sciuro-hypnum glaciale (Schimp.) Ignatov \& Huttunen (=Brachythecium } \\
\text { glaciale Schimp) }\end{array}$ & & $\mathrm{X}$ & & \\
\hline \multicolumn{5}{|l|}{ BRYACEAE } \\
\hline Bryum archangelicum Bruch \& Schimp (=Bryum amblyodon Müll. Hal.) & $\mathrm{X}$ & $\mathrm{X}$ & $\mathrm{X}$ & $\mathrm{X}$ \\
\hline Bryum argenteum Hedw. & $\mathrm{X}$ & $\mathrm{X}$ & $\mathrm{X}$ & $\mathrm{X}$ \\
\hline $\begin{array}{l}\text { Gemmabryum dichotomum }(\text { Hedw.) J. R. Spence \& H. P. Ramsay } \\
\text { (=Bryum dichotomum Hedw.) }\end{array}$ & $\mathrm{X}$ & $\mathrm{X}$ & & $\mathrm{X}$ \\
\hline Bryum orbiculatifolium Cardot \& Broth. & $\mathrm{X}$ & $\mathrm{X}$ & $\mathrm{X}$ & \\
\hline Bryum pallescens Schleich. ex Schwaegr. & & $\mathrm{X}$ & & $\mathrm{X}$ \\
\hline $\begin{array}{l}\text { Ptychostomum pseudotriquetrum (Hedw.) J. R. Spence \& H. P. Ramsay } \\
\text { [=Bryum pseudotriquetrum (Hedw.) P. Gaertn., B. Mey. \& Scherb.] }\end{array}$ & $\mathrm{X}$ & $\mathrm{X}$ & $\mathrm{X}$ & $\mathrm{X}$ \\
\hline Pohlia cruda (Hedw.) Lindb. & $\mathrm{X}$ & $\mathrm{X}$ & $\mathrm{X}$ & \\
\hline Pohlia drummondii (Müll. Hal.) A. L. Andrews & $\mathrm{X}$ & $\mathrm{X}$ & $\mathrm{X}$ & \\
\hline Pohlia nutans (Hedw.) Lindb. & $\mathrm{X}$ & $\mathrm{X}$ & & \\
\hline Pohlia wahlenbergii (F. Weber \& D. Mohr) A.L. Andrews & $\mathrm{X}$ & $\mathrm{X}$ & & \\
\hline \multicolumn{5}{|l|}{ DICRANACEAE } \\
\hline $\begin{array}{l}\text { Dicranella campylophylla (Taylor) A. Jaeger [=Anisothecium cardotii (R. } \\
\text { Br. bis.) Ochyra] }\end{array}$ & & $\mathrm{X}$ & & \\
\hline Chorisodontium aciphyllum (Hook. f. \& Wills.) Broth. & $\mathrm{X}$ & $\mathrm{X}$ & $\mathrm{X}$ & $\mathrm{X}$ \\
\hline $\begin{array}{l}\text { Kiaeria pumila (Mitt.) Ochyra } \\
\text { DITRICHACEAE }\end{array}$ & & $\mathrm{X}$ & & \\
\hline Ceratodon purpureus (Hedw.) Brid. & $\mathrm{X}$ & $\mathrm{X}$ & $\mathrm{X}$ & $\mathrm{x}$ \\
\hline Distichium capillaceum (Hedw.) Bruch \& Schimp & $\mathrm{X}$ & $\mathrm{X}$ & $\mathrm{X}$ & $\mathrm{X}$ \\
\hline Ditrichum hyalinum (Mitt.) Kuntze & & $\mathrm{X}$ & & \\
\hline Ditrichum hyalinocuspidatum Cardot (=Ditrichum lewis-smithii Ochyra) & & $\mathrm{X}$ & & \\
\hline \multicolumn{5}{|l|}{ ENCALYPTACEAE } \\
\hline Encalypta rhaptocarpa Schwaegr. & $\mathrm{X}$ & $\mathrm{X}$ & $\mathrm{X}$ & $\mathrm{X}$ \\
\hline \multicolumn{5}{|l|}{ GRIMMIACEAE } \\
\hline Orthogrimmia sessitana (De Not.) Ochyra \& Zarnowiec & & $\mathrm{X}$ & & $\mathrm{X}$ \\
\hline Bucklandiella sudetica (Funck) Bednarek-Ochyra \& Ochyra & & $\mathrm{X}$ & & \\
\hline Schistidium amblyophyllum (Müll. Hal.) Ochyra \& Hertel & & $\mathrm{X}$ & & \\
\hline Schistidium antarctici (Cardot) L. I. Savicz \& Smirnova & & $\mathrm{X}$ & & \\
\hline Schistidium cupulare (Müll. Hal.) Ochyra & & $\mathrm{X}$ & & \\
\hline Schistidium falcatum (Hook. f. \& Wils.) B. Bremer & & $\mathrm{X}$ & & \\
\hline Schistidium halinae Ochyra & & $\mathrm{X}$ & $\mathrm{X}$ & $\mathrm{X}$ \\
\hline $\begin{array}{l}\text { Schistidium andinum (Mitt.) Herzog [=Schistidium occultum (Müll. Hal.) } \\
\text { Ochyra \& Matteri] }\end{array}$ & & & $\mathrm{X}$ & \\
\hline Schistidium rivulare (Brid.) Pobp. & & $\mathrm{X}$ & & \\
\hline Schistidium steerei Ochyra & & $\mathrm{X}$ & & \\
\hline Schistidium urnulaceum (Müll. Hal.) B. G. Bell. & & $\mathrm{X}$ & & \\
\hline
\end{tabular}


TABLE I (continuation)

\begin{tabular}{|c|c|c|c|c|}
\hline MOSSES & Elephant & $\begin{array}{c}\text { King } \\
\text { George }\end{array}$ & Nelson & Deception \\
\hline \multicolumn{5}{|l|}{ HYPNACEAE } \\
\hline Hypnum revolutum (Mitt.) Lindb. & & $\mathrm{X}$ & $\mathrm{X}$ & \\
\hline Platydictya jungermannioides (Brid.) H. A.Crum & & $\mathrm{X}$ & & \\
\hline \multicolumn{5}{|l|}{ MEESIACEAE } \\
\hline Meesia uliginosa Hedw. & & $\mathrm{X}$ & & \\
\hline \multicolumn{5}{|l|}{ ORTHOTRICHACEAE } \\
\hline Muelleriella crassifolia (Hook. f. \& Wils.) Dusén & $\mathrm{X}$ & $\mathrm{X}$ & & \\
\hline \multicolumn{5}{|l|}{ POLYTRICHACEAE } \\
\hline Notoligotrichum trichodon (Hook. \& Wilson) G. L. Sm. & & $\mathrm{X}$ & & \\
\hline Polytrichastrum alpinum (Hedw.) G. L. Smith & $\mathrm{X}$ & $\mathrm{X}$ & $\mathrm{X}$ & $\mathrm{X}$ \\
\hline Polytrichum strictum Menzies ex Brid. & $\mathrm{X}$ & $\mathrm{X}$ & $\mathrm{X}$ & \\
\hline Polytrichum juniperinum Hedw. & $\mathrm{X}$ & $\mathrm{X}$ & $\mathrm{X}$ & $\mathrm{X}$ \\
\hline Polytrichum piliferum Hedw. & $\mathrm{X}$ & $\mathrm{X}$ & $\mathrm{X}$ & $\mathrm{X}$ \\
\hline \multicolumn{5}{|l|}{ POTTIACEAE } \\
\hline Didymodon brachyphyllus (Sull.) R. H. Zander & $\mathrm{X}$ & $\mathrm{X}$ & & \\
\hline Hennediella antarctica (Angström) Ochyra \& Matteri & $\mathrm{X}$ & $\mathrm{X}$ & $\mathrm{X}$ & $\mathrm{X}$ \\
\hline Hennediella heimii (Hedw.) R. H. Zander. & $\mathrm{X}$ & $\mathrm{X}$ & $\mathrm{X}$ & $\mathrm{X}$ \\
\hline Stegonia latifolia (Schwaegr.) Vent ex Broth. & & $\mathrm{X}$ & & $\mathrm{X}$ \\
\hline Syntrichia filaris (Müll. Hal.) R. H. Zander & $\mathrm{X}$ & $\mathrm{X}$ & & $\mathrm{X}$ \\
\hline $\begin{array}{l}\text { Syntrichia magellanica (Mont.) R.H. Zander [=Syntrichia princeps (De } \\
\text { Not.) Mitt.] }\end{array}$ & $\mathrm{X}$ & $\mathrm{X}$ & $\mathrm{X}$ & $\mathrm{X}$ \\
\hline Syntrichia saxicola (Cardot) R. H. Zander & $\mathrm{X}$ & $\mathrm{X}$ & $\mathrm{X}$ & $\mathrm{X}$ \\
\hline \multicolumn{5}{|l|}{ SELIGERACEAE } \\
\hline Hymenoloma crispulum (Hedw.) Ochyra & & $\mathrm{X}$ & & $\mathrm{X}$ \\
\hline Hymenoloma grimmiaceum (Müll. Hal.) Ochyra & $\mathrm{X}$ & $\mathrm{X}$ & $\mathrm{X}$ & \\
\hline Hymenoloma antarcticum (Müll. Hal.) Ochyra & $\mathrm{X}$ & $\mathrm{X}$ & $\mathrm{X}$ & $\mathrm{X}$ \\
\hline Holodontium strictum (Hook f. \& Wilson) Ochyra & & $\mathrm{X}$ & & \\
\hline Syntrichia saxicola (Cardot) R. H. Zander & $X$ & $\mathrm{X}$ & $\mathrm{X}$ & $\mathrm{X}$ \\
\hline \multicolumn{5}{|l|}{ SELIGERACEAE } \\
\hline Hymenoloma crispulum (Hedw.) Ochyra & & $\mathrm{X}$ & & $\mathrm{X}$ \\
\hline Hymenoloma grimmiaceum (Müll. Hal.) Ochyra & $\mathrm{X}$ & $\mathrm{X}$ & $\mathrm{X}$ & \\
\hline Hymenoloma antarcticum (Müll. Hal.) Ochyra & $\mathrm{X}$ & $\mathrm{X}$ & $\mathrm{X}$ & $\mathrm{X}$ \\
\hline Holodontium strictum (Hook f. \& Wilson) Ochyra & & $\mathrm{X}$ & & \\
\hline
\end{tabular}

Comparing the data on the vegetal communities of different areas, we can see that Stinker Point has the greater Bryum argenteum and Chorisodontium acyphyllum formations, which are reduced in the other areas (Pereira and Putzke 1994). At Rip Point the mosses are dispersed over all the area, in small patches or cushions, rarely carpets, and the lakes rich in submerged communities (Putzke and Pereira 1998a, b). The biggest lake in Elephant Island also has a submerged community that is, not observed on the Keller Peninsula (Pereira et al. 2007). The 
vegetation of Deception Island (Figure 2) is still recovering the last volcanic eruption, but some fast growing patches of $D$. antarctica are found. For description of the main plants communities see Pereira et al. 2008 and Victoria et al. 2009b.

On Elephant and Nelson Islands human impact are minimal since the only buildings present are small refuges that are only sporadically occupied (Pereira and Putzke 1994, Puztke and Pereira 1998b). On the first island, the Brazilian refugee Wiltgen was completely removed, contributing to the moss regeneration at Stinker Point.

On Deception Island the touristic activity has been affecting the flora, but new rules are being followed and the impact is being reduced.

Keller Peninsula studies over the years have shown that human activity impacts the moss formations in a negative manner, but in some areas the plant communities increase faster (Pereira et al. 2007, 2008).

The use of land plants for nest building was studied in Catharacta spp, in L. dominicanus and in Phalacrocorax atriceps. In the three cases studied land plant were the principal material in the nest, but lots of garbage are being used (Pereira et al. 1990a, b, Sander et al. 2004).

Plant checklist from the areas studied, collected and indentified by Brazilian researchers and preserve in herbarium

\section{ANGIOSPERMAE}

POACEAE

Deschampsia antarctica Desv.

CARYOPHYLLACEAE

Colobanthus quitensis (Kunth) Bartl.

\section{LIVERWOTS}

Cephaloziella varians (Gottsche) Stephani

Lophozia excisa (Dicks.) Dumort.

Marchantia berteroana Lehm. \& Lindenb.

\section{MOSSES}

AMBLYSTEGIACEAE

Cratoneuropsis relaxa ssp. minor

Sanionia uncinata (Hedw.) Loeske

Sanionia georgico-uncinata (Müll Hal.) Ochyra \& Hedenas

Warnstorfia laculosa (Müll. Hal.) Ochyra \& Matteri

Warnstorfia sarmentosa (Wahlenb.) Hedenas

ANDREAEACEAE

Andreaea depressinervis Cardot

Andreaea gainii Cardot

Andreaea regularis Müll. Hal.

BARTRAMIACEAE

Bartramia patens Brid.

Conostomum magellanicum Sull.

BRACHYTHECIACEAE

Brachythecium austrosalebrosum (Müll. Hal.) Kindb. Sciuro-hypnum glaciale (Schimp.) Ignatov \& Huttunen

BRYACEAE

Bryum archangelicum Bruch \& Schimp

Bryum argenteum Hedw.

Bryum orbiculatifolium Cardot \& Broth.

Bryum pallescens Schleich. ex Schwaegr.

Ptychostomum pseudotriquetrum (Hedw.) J. R. Spence \& H. P.

Pohlia cruda (Hedw.) Lindb.

Pohlia drummondii (Müll. Hal.) A. L. Andrews in Grout

Pohlia nutans (Hedw.) Lindb.

Pohlia wahlenbergii (F. Weber \& D. Mohr) A.L. Andrews

\section{DICRANACEAE}

Chorisodontium aciphyllum (Hook. f. \& Wills.) Broth.

Dicranella campylophylla (Taylor) A. Jaeger

Dicranella varia (Hedw.) Schimp

Kiaeria pumila (Mitt. \& Hook. f.) Ochyra - very rare.

DITRICHACEAE

Ceratodon purpureus (Hedw.) Brid. 
Distichium capillaceum (Hedw.) Bruch \& Schimp Ditrichum hyalinum (Mitt.) Kuntze

Ditrichum hyalinocuspidatum Cardot

ENCALYPTACEAE

Encalypta rhaptocarpa Schwaegr.

GRIMMIACEAE

Grimmia reflexidens Müll. Hal.

Bucklandiella sudetica (Funck) Bednarek-Ochyra \& Ochyra

Schistidium amblyophyllum (Müll. Hal.) Ochyra \& Hertel

Schistidium antactici (Cardot) L. I. Savicz \& Smirnova

Schistidium cupulare (Müll. Hal.) Ochyra

Schistidium falcatum (Hook. f. \& Wils.) B. Bremer Schistidium halinae Ochyra

Schistidium andinum (Mitt.) Herzog Schistidium rivulare (Brid.) Pobp.

Schistidium steerei Ochyra

Schistidium urnulaceum (Müll. Hal.) B. G. Bell.

HYPNACEAE

Hypnum revolutum (Mitt.) Lindb.

Platydictya jungermannioides (Brid.) Crum

MEESIACEAE

Meesia uliginosa Hedw.

ORTHOTRICHACEAE

Muelleriella crassifolia (Hook. f. \& Wils.) Dusén

POLYTRICHACEAE

Polytrichastrum alpinum (Hedw.) G. L. Smith

Polytrichum strictum Brid.

Polytrichum juniperinum Hedw.

Polytrichum piliferum Hedw.

POTTIACEAE

Didymodon brachyphyllus (Sull.) R. H. Zander

Hennediella antarctica (Angstr.) Ochyra \& Matteri

Hennediella heimii (Hedw.) Zand.

Stegonia latifolia (Schwaegr.) Vent ex Broth.

Syntrichia filaris (Müll. Hal.) Zand.

Syntrichia magellanica (Mont.) R.H. Zander
Syntrichia saxicola (Cardot) Zand.

\section{SELIGERACEAE}

Hymenoloma antarcticum (Müll. Hal.) Ochyra

Hymenoloma crispulum (Hedw.) Ochyra

Hymenoloma grimmiaceum (Müll. Hal.) Ochyra

\section{ALGAE}

MACROSCOPIC CONTINENTAL ALGAE

Prasiola crispa (Lightfoot) Menegh

\section{MACROSCOPIC FUNGI}

Lamprospora miniatopsis Spooner

Omphalina antarctica Sing.

Galerina moelleri Bas.

Galerina perara Sing.

\section{LICHENIZED AND LICHENICOLOUS FUNGI}

Acarospora macrocyclos Vain.

Amandinea coniops (Wahlenb.) M. Choisy ex Scheid. \& H. Mayrhofer. (=Buellia coniops)

Amandinea petermanii (Hue) Matzer, H. Mayrhofer $\&$ Scheid. [=Rinodina petermanii (Hue) Darb.]

Arthopyrenia maritima Øvstedal

Arthrorhaphis citrinella (Ach.) Poelt

Austrolecia antarctica Hertel

Bacidia stipata Lamb

Biatorella antarctica B. J. Murray

Bryonora castanea (Hepp) Poelt

Bryoria chalybeiformis (L.) Brodo et D. Hawksw.

Buellia anisomera Vain.

Buellia augusta Vain.

Buellia cladocarpiza Lamb

Buellia granulosa (Darb.) Dodge

Buellia latemarginata Darb.

Buellia nelsonii Darb.

Buellia papillata (Sommerf.) Tuck.

Buellia perlata (Hue) Darb.

Buellia pycnogonoides Darb.

Buellia russa (Hue) Darb.

Buellia subpedicillata (Hue) Darb.

Caloplaca amniospila (Ach.) H. Olivier

Caloplaca athallina Darb. 
Caloplaca buelliae Olech \& Søchting

Caloplaca cirrochrooides (Vain.) Zahlbr.

Caloplaca citrina (Hoffm.) Th. Fr.

Caloplaca iomma Olech \& Søchting

Caloplaca millegrana (Müll. Arg.) Zahlbr.

Caloplaca psoromatis Olech \& Søchting

Caloplaca regalis (Vain.) Zahlbr.

Caloplaca siphonospora Olech \& Søchting

Caloplaca sublobulata (Vain.) Zahlbr.

Caloplaca tetraspora (Nyl.) H. Oliv.

Caloplaca tiroliensis Zahlbr.

Candelaria murrayi (Dodge) Poelt

Candelariella hallettensis (Murray) Øvstedal

Candelariella vitellina (Hoffm.) Müll. Arg.

Carbonea assetiens (Nyl.) Hertel

Carbonea vorticosa (Flörke) Hertel

Catapyrenium daedaleum (Kremp.) Stein

Catapyrenium lachneum (Ach.) R. Sant.

Catillaria corymbosa (Hue) Lamb

Cetraria aculeata (Schreb.) Fr. [=Coelocaulon

aculeatum (Schreber) Link]

Cladonia cariosa (Ach.) Spreng.

Cladonia chlorophaea (Flörke ex Sommerf.)

Spreng.

Cladonia furcata (Huds.) Schrader

Cladonia gracilis (L.) Willd.

Cladonia metacorallifera Asah.

Cladonia phyllophora Ehrh. ex Hoffm.

Cladonia pyxidata (L.) Hoffm.

Cladonia rangiferina (L.) Weber

Coleopogon epiphorellus (Nyl.) Brusse \& Kärnefelt

[=Coelocaulon epiphorellum (Nyl) Kärnef.]

Cystocoleus ebeneus (Dillwyn) Thwaites

Dermatocarpon intestiniforme (Körb.) Hasse

Haematomma erythroma (Nyl.) Zahlbr.

Himantormia lugubris (Hue) Lamb

Huea cerussata (Hue) C.W. Dodge \& G.E. Baker

Huea coralligera (Hue) C.W. Dodge \& G.E. Baker

Hypogymnia lugubris (Pers.) Krog

Hypogymnia lububris var. compactior (Zahlbr.) D.

C. Linds.

Japewia tornoensis (Nyl.) Tønsberg
Lecania brialmontii (Vain.) Zahlbr.

Lecania gerlachei (Vain.) Zahlbr.

Lecanora dispersa (Pers.) Sommerf.

Lecanora expectans Darb.

Lecanora mons-nivis Darb.

Lecanora physciella (Darb.) Hertel

Lecanora polytropa (Hoffm.) Rabenh.

Lecidea atrobrunnea (Ramond ex Lam. et DC.)

Schaer.

Lecidea lapicida (Ach.) Ach.

Lecidea sarcogynoides Körb.

Lecidea sciatrapha Hue

Lecidella stigmatea (Ach.) Hertel and Leuckert

Lecidella wulfenii (Hepp) Körb.

Leptogium menziesii Mont.

Leptogium puberulum Hue

Massalongia carnosa (Dicks.) Körb.

Mastodia tesselata (Hook.f. \& Harv.) Hook.f. \& Harvey

Megaspora verrucosa (Ach.) Hafellner

Micarea assimilata (Nyl.) Coppins (=Lecidea assimilata Nyl.)

Microglaena antarctica Lamb

Ochrolechia frigida (Sw.) Lynge

Ochrolechia parella (L.) A. Massal.

Pannaria hookeri (Borrer ex Sm.) Nyl.

Parmelia saxatilis (L.) Ach.

Physcia caesia (Hoffm.) Fürnr.

Physcia dubia (Hoffm.) Lettau

Physconia muscigena (Ach.) Poelt

Placopsis contortuplicata Lamb

Poeltidea perusta (Nyl.) Hertel et Hafellner

Polyblastia gothica Th. Fr.

Porpidia albocaerulescens (Wulfen) Hertel et

Knoph

Porpidia crustulata (Ach.) Hertel et knoph

Pseudephebe minuscula (Nyl. ex Arnold) Brodo et

D. Hawksw. (=Alectoria minuscula Lindsay)

Pseudephebe pubescens (L.) Choisy

Psoroma hypnorum (Vahl) Gray

Ramalina terebrata Hook et Tayl.

Rhizocarpon geminatum Körb. 
Rhizocarpon geographicum (L.) DC.

Rhizocarpon polycarpon (Hepp) Th. Fr.

Rhizoplaca aspidophora (Vain.) Redón

Rhizoplaca melanophthalma (DC. in Lam. et DC.)

Leuck. et Poelt

Rinodina deceptionis Lamb

Rinodina mniaraea (Ach.) Körb.

Rinodina turfacea (Wahlenb.) Körb.

Sphaeorophorus fragilis (L.) Pers.

Sphaeorophorus globosus (Hudson) Vain.

Sphaeorophorus melanocarpus (Sw.) DC.

Staurothele gelida (Hook \& Tayl.) Lamb

Stereocaulon alpinum Laurer ex Funck

Stereocaulon glabrum (Müll. Arg.) Vain.

Tephromela atra (Hudson) Hafellner

Thelocarpon cyaneum Olech et Alstrup

Tremolecia atrata (Ach.) Hertel

Umbilicaria aprina Nyl.

Umbilicaria decussata (Vill.) Zahlbr.

Umbilicaria propagulifera (Vain.) Llano

Umbilicaria rufidula (Hue) Filson

Usnea acromelana Stirton

Usnea antarctica Du Rietz

Usnea aurantiacoatra (Jacq.) Bory

Usnea trachycarpa (Stirt.) Mull. Arg.

Verrucaria ceuthocarpa Wahlenb.

Verrucaria cylindrophora Vain.

Verrucaria dispartita Vain.

Verrucaria elaeoplaca Vain.

Verrucaria halizoa Leight.

Verrucaria psycrophila Lamb

Verrucaria racovitzae Vain.

Verrucaria tesselatula Nyl.

Xanthoria candelaria (L.) Th. Fr.

Xanthoria elegans (Link.) Th. Fr.

Actually, the main concern of the Brazilian botanists in Antarctica is to describe and map the plant and microbial community in order to better understand the processes that drive in the Antarctic ice-free areas, and to relate these to human impacts or natural phenomena. Global changes are leading to the loss of ice cover in Antarctica, and with it, potentially dramatic changes in plant and microbial community composition. There is an urgent need to establish georeferenced baseline maps of plant and soil microbial distribution in order to evaluate future compositional changes through the years. Brazilian researchers have already began this important work, contributing to the management and monitoring studies in the Antarctic ice free areas (Pereira and Putzke 1994, Pereira et al. 2007, 2008, 2009, Putzke and Pereira 1998b, Victoria et al. 2004, 2006, 2009a, b, Victoria and Pereira 2007).

\section{CONCLUSIONS}

In twenty-five years of work by botanists from the Brazilian Antarctic Program investigating the plant communities in Antarctic ice free areas, six papers on the chemistry of lichens, six papers on plant taxonomy, five papers on plant biology, two works on UVB photoprotection and eleven papers on plant communities were published.

At the present, Brazilian botanists are studying the plant communities in Antarctic ice free areas in order to understand their relationship to soil microbial communities, their biodiversity, the distribution of the plants populations and their relationship with birds colonies. Baseline maps of plant communities and their distribution are being made in order to arguments studies of their development and environmental impacts in the future. In addition to these activities, a group of Brazilian researchers are undertaking studies related to Antarctic plant genetic diversity, plant chemistry and the biotechnological applications of these. It is believed that knowledge of the biotechnological potentials of Antarctic plants, in addition to research on plant/communities biology and evolving processes is essential to the valuation and therefore the preservation of these natural resources. 


\section{ACKNOWLEDGMENTS}

This work was supported by the Brazilian Antarctic Program through the Conselho Nacional de Desenvolvimento Científico e Tecnológico (CNPq process no. 574018/2008), Fundação de Amparo à Pesquisa do Estado do Rio de Janeiro (FAPERJ process E-26/170.023/2008), Ministry of Environment - MMA, Ministry of Science, Technology and Innovation - MCTI and CIRM.

\section{RESUMO}

Este trabalho tem como objetivo resumir os resultados das pesquisas realizadas por brasileiros nas comunidades vegetais de áreas livres de gelo da Antártida, durante os últimos vinte e cinco anos. Tais investigações iniciaram em 1988, sendo realizados nas ilhas Elefante, Rei George, Nelson de Deception. Durante este período foram publicados seis trabalhos sobre a química de líquens, sete sobre taxonomia vegetal, cinco sobre a biologia de plantas, dois sobre fotoproteção UVB, três sobre as relações entre as comunidades vegetais e colônias de aves e onze sobre as comunidades de plantas de áreas de degelo, abordando biogeografia e fitossociologia. Atualmente, os botânicos brasileiros estão pesquisando as comunidades vegetais de áreas de degelo da Antártica, buscando entender suas relações com as comunidades microbianas do solo, a biodiversidade, a distribuição das populações de plantas e sua relação com as colónias de aves. Além dessas atividades, um grupo de pesquisadores está investigando a diversidade genética vegetal através de estudos de genética de populações e a bioquímica das plantas.

Palavras-chave: Produção científica, Botânica, Shetlands do Sul, Antarctica.

\section{REFERENCES}

Czeczuga B AND XAVIER-Filho L. 1987. Investigations carotenoids in Lichens x Luteoxanthin and Apo - JQ Violaxanthal in Lichens from the Antarctica. Ser Cient INACH 36: 151-155.

CZECZUGA B AND XAVIER-FILHO L. 1988. Investigations on caratenoids in Embryophyta X. I. The presence of torulene in mosses from the Antarctica. Ser Cient INACH 37: 111-116.
Elster J, Degma P, KovÁcik L, VAlentovÁ L, SRANKovÁ K AND PEREIRA AB. 2008. Freezing and deccication injury resistance in the filamentous green alga Klebsormidium from the Antarctuc, Arctic and Slovakia. Versita 63(6): 843-851.

GonÇALVes PN, Neves PCP, TONIn A AND PEREIRA AB. 2008 Morfologia dos grãos de pólen de angiospermas modernas da Ilha King George, Ilhas Shetlands do Sul, Península Antártica. Gaea Heidelbergensis 4(1): 24-31.

Kappel Pereira B, Pereira AB, Groff A, SchroderPfEIFER NT AND SILVA J. 2004. Avaliação do efeito fotoprotetivo de Polytrichum juniperinum Hedw., Colobanthus quitensis (Kunth.) Bartl. e Deschampsia antarctica Desv., através de Ensaio Cometa em Helix aspersa (Mülller, 1774). In: Actas do V Simposio Argwentino e I latinoamericano sobre Investigaciones Antarticas. Buenos Aires - Argentina. IAA 1: 1-4.

Kappel Pereira B ET AL. 2009. Protective effects of three extracts from Antarctic plants against ultraviolet radiation in several biological models. J Photoch Photobio B 96: $117-129$.

KovÁcIK L, Gianluppi L, HindÁKOvÁ A AND PEREIRA AB. 2004. Diatoms from Keller Peninsula, King George Island, Antarctica - preliminary Study. Bull Slov Bot Spolocn 10: 74-76.

KovÁCIK L AND Pereira AB. 2001. Green alga Prasiola crispa and its lichenized form Mastodia tesselata in Antarctic environment: General aspects. Nova Hedwigia 123: 465478.

Martins MFN, Spielmann AA, PutzKe J AND Pereira AB. 2004. Lichenized Fungi on Men-made Substrata in Deception Island, South Shetlands, Antarctica. In: Actas del V Simposio Argentino y I Latinoamericano de Investigaciones Antarticas. Buenos Aires - Argentina. IAA 1: 1-4.

OCHYRA R, LEWIS SMITH RI AND BEDNAREK-OCHYRA H 2008. The illustrated moss flora of Antarctica. Cambridge University Press. Cambridge, 685 p.

OLECH M. 1996. Human impact on terrestrial ecosystems in west Anytarctica. Proceed NIPR Symp Polar Biol 9: 299306.

ØvSTEDAL DO AND LEWIS SMITH RI. 2001. Lichens of Antarctica and South Georgia - A guide to their identification and ecology. Studies in Polar Research. Cambridge University Press, $411 \mathrm{p}$.

Øvstedal DO AND LewIS SMITH RI. 2004. Additions and corrections to the lichens of Antarctica and South Georgia. Cryptogamie. Mycologie 25: 323-331

ØvSTEDAL DO AND LEWIS SMITH RI. 2009. Further additions to the lichen flora of Antarctica and South Georgia, Nova Hedwigia 88: 157-168.

PEREIRA AB. 1990. A new ocurrence of Usnea in Antarctica. Pesq Antártica Bras 2: 13-16.

PereIRA AB. 2004. Comunidades Vegetais de Áreas de Degelo da Antártica Marítima. In: Anais do Congresso Nacional de Botânica 1, p. 1-15. 
Pereira AB, Francelino MR AND Roesch LFW. 2009. Vegetal communities from ice-free areas of Copacabana, King George Island, Antarctica. Ann Act Rep 2009: 25-26.

Pereira AB And Putzke J. 1994. Floristic Composition of Stinker Point, Elephant Island, Antarctica. Korean Journal of polar Research. Korean 5(2): 37-47.

Pereira AB, PutzKe ML And PutzKe J. 2008. Biological Communities of Keller Peninsula, King George Island Antarctica. Cad Pesqui Ser Biol 20(3): 63-74.

Pereira AB, SAnder M And PutzKe J. 1990b. Plants utilized by Larus dominicanus Lichtenstein, 1823 for nest building et the South Shetland Island, Antarctica. Pesq Antártica Bras 2: 79-85.

Pereira AB, Sander M and Strieder M. 1990a. Biologic notes about Turret Point, King George Island, Antarctica. Pesq Antártica Bras 2: 73-77.

Pereira AB, Spielmann AA, Martins MFN AND FRANCELINO MR. 2007. Plant Communities from ice-free areas of Keller Peninsula, King George Island, Antarctica. Oecol Bras 10(1): 14-22.

PutzKe J AND Pereira AB. 1990. Mosses of King George Island, Antarctica. Pesq Antártica Bras 2: 17-71.

Putzke J And Pereira AB. 1996. Macroscopic Fungi From The South Shetlands, Antarctica. Ser Cient INACH 46: 31-39.

Putzke J AND Pereira AB. 1998a. The lichens of Rip Point, Nelson Island, Antarctica. Pesq Antártica Bras 3(1): 87-101.

Putzke J AND Pereira AB. 1998b. Mosses communities of Rip Point in Northern Nelson Island, Antarctica. Pesq Antártica Bras 3(1): 104-115.

Putzke J AND Pereira AB. 2001. The Antarctic Mosses with special reference to the South Shetland Islands. $1^{\text {st }}$ ed., Canoas, Rio Grande do Sul, Brasil, EDULBRA, 186 p.

Putzke J, Pereira AB And Putzke MTL. 2004. New Record of Myxomycetes to the Antarctica. In: Actas del V Simposio Argentino y I Latinoamericano de Investigaciones Antarticas. Buenos Aires - Argentina. IAA 1: 1-4.

Sander M, Costa ES, Santos CR And Pereira AB. 2004. Colônia de Aves e Comunidades Vegetais da Península Keller, Ilha Rei George (25 de Mayo), Antártica. In: Actas Del V Sinposio Argentino y I Latinoamericano de Investigaciones Antarticastas del. Buenos Aires Argentina. IAA 1: 1-4.

SouZA JEB. 2008. Brasil na Antártica - 25 anos de história. São Carlos, SP, Vento Verde Editora, 167 p.

SPENCE JR. 2005. New genera and combinations in Bryaceae (Bryales, Musci) for North America. Phytologia 87(1): 15-28.
SPENCE JR. 2007. Nomenclatural changes in the Bryaceae (Bryopsida) for North America II. Phytologia 89(1): 110-114.

SRAmKova K, KovÁciK L, JANCUSOVA M, Kristin J AND PEREIRA AB. 2007. Diatoms from Whalers Bay of Deception Island, South Shetlands Antarctica preliminary study. Bull Slov Bot Spol 29: 17-20.

TuRner J And Marshall GJ. 2011. Climate Change in the Polar Regions. Cambridge, UK, Cambridge University Press, $434 \mathrm{p}$.

Victoria FC, Albuquerque MP AND Pereira AB. 2006. Lichen-Moss asociations in plant communiyies of the Southwest Admiralty Bay, King George Island, Antarctica. Neotropical Biol Conserv 1: 84-89.

Victoria FC, Costa DP AND Pereira AB. 2004. Characterization of Plant Communities in Ice-free areas Adjoining the Polish Station H. Arctowski, Admiralty Bay, King George Island, Antarctica. In: Actas del V Simposio Argentino y 1 Latinoamericano sobre Investigaciones Antarticas. Buenosd Aires - Argentina. IAA 1: 1-4.

VICTORIA FC, COSTA DP AND PEREIRA AB. 2009a. Life-forms of moss species in defrosting areas of King George Island, South Shetland Islands, Antarctica. Bioscience Journal 25(3): 151-160.

VICTORIA FC AND PEREIRA AB. 2007. Índice de valor ecológico (IES) como ferramenta para estudos fitossociológicos e conservação das espécies de musgos na Baia do Almirantado, Ilha Rei George, Antártica Marítima. Oecol Bras 11: 50-55.

VICTORIA FC, PEREIRA AB AND COSTA DP. 2009b. Composition and distribution of moss formation in the ice-free areas adjoining the Arctowski region, Admiralty Bay, King George Insland, Antarctica. Iheringia Ser Bot 64:81-91.

XAVIER-FILHO L, GUZMAN GG AND RUdOLPH ED. 1989. Observations on nutrient flow in a maritime Antartic Tundra Community. Bol Soc Broter 62: 97-106.

Xavier-Filho L, KolakawsKa AC AND Vicente C. 1986. Beta-caratenoid composition of Caloplaca regalis (Vain) Zahbr. (Lickens) and of penguin faces at king George Island (Antartica). Pol Polar Res 7(3): 289-294.

XAVIER-FILHo L, LEITE BM AND OliveIRA E. 1990. Atividade antimicrobiana de Liquens Antártios. Bol Soc Brot 63: 93-98.

XaVier-Filho L, Pereira C, PAUlo MQ AND Silva YO. 1988. Organismos da Tundra Antártica e a química de Produtos Naturais. An Acad Cienc do Estado de São Paulo Perspect Quim Prod 1: 27-48. 